\title{
Long Range Bond-Bond Correlations in Dense Polymer Solutions
}

\author{
J.P. Wittmer, ${ }^{1}$, 因H. Meyer, ${ }^{1}$ J. Baschnagel,${ }^{1}$ A. Johner, ${ }^{1}$ S. Obukhov, ${ }^{1,2}$ L. Mattioni, ${ }^{3}$ M. Müller ${ }^{4}$ and A.N. Semenov ${ }^{1}$ \\ ${ }^{1}$ Institut Charles Sadron, 6 Rue Boussingault, 67083 Strasbourg, France \\ ${ }^{2}$ Department of Physics, University of Florida, Gainesville FL 32611, USA \\ ${ }^{3}$ LPMCN, Université Claude Bernard \& CNRS, Lyon, France \\ ${ }^{4}$ Institut für Physik, Johannes Gutenberg-Universität, Staudinger Weg 7, D-55099 Mainz, Germany
}

(Dated: November 19, 2018)

\begin{abstract}
The scaling of the bond-bond correlation function $P_{1}(s)$ along linear polymer chains is investigated with respect to the curvilinear distance, $s$, along the flexible chain and the monomer density, $\rho$, via Monte Carlo and molecular dynamics simulations. Surprisingly, the correlations in dense three dimensional solutions are found to decay with a power law $P_{1}(s) \sim s^{-\omega}$ with $\omega=3 / 2$ and the exponential behavior commonly assumed is clearly ruled out for long chains. In semidilute solutions, the density dependent scaling of $P_{1}(s) \approx g^{-\omega_{0}}(s / g)^{-\omega}$ with $\omega_{0}=2-2 \nu=0.824(\nu=0.588$ being Flory's exponent) is set by the number of monomers $g(\rho)$ contained in an excluded volume blob of size $\xi$. Our computational findings compare well with simple scaling arguments and perturbation calculation. The power-law behavior is due to self-interactions of chains caused by the chain connectivity and the incompressibility of the melt. This study suggests a careful reexamination of the operational definitions used for the experimental determination of the persistence length.
\end{abstract}

PACS numbers: 05.40.Fb, 05.10.Ln, 61.25.Hq

In this Letter we study the correlations of the directions of bonds along polymer chains in semidilute solutions and melts [1, 2, 3]. We focus on flexible monodisperse chains of $N$ monomers (cf. Fig. 1) under good solvent conditions in three dimensions $(d=3)$ where both the bond length $l$ and the excluded volume screening length $\xi[2,4]$ are always much smaller than the chain end-to-end distance $R_{e}$. In principle, once the bond-bond correlations are computed all other conformational single chain properties can be derived. Importantly, being the (second) derivative of the spatial distances along the chains, they allow us to probe directly — without trivial ideal contributions - the non-gaussian corrections proposed recently [5]. As we shall see, these corrections are crucial to make the description of dense polymer systems, first proposed by Flory [3] and later corroborated by Edwards [2, 4], fully self-consistent.

The bond-bond correlation function $P_{1}(s)$ is generally believed to decrease exponentially [3] . This belief is based on the few simple single chain models which have been solved rigorously [3, 6] and on the assumption that all long range interactions are negligible on distances larger than $\xi$ due to the screening mechanism described by Edwards [2, 4]. Hence, only correlations along the backbone of the chains are expected to matter and it is then straightforward to work out that an exponential cut-off is inevitable due to the multiplicative loss of any information transferred recursively along the chain [3].

We demonstrate here that this assumption is in fact incorrect and that unexpected long range correlations remain. They are responsible for a scale free power law regime with $P_{1}(s)=c_{a}(\rho) s^{-\omega}$ for $g(\rho) \ll s \ll N(g(\rho)$ being the number of monomers per blob at monomer density $\rho$ ) characterized by an exponent $\omega>1$ and a density dependent amplitude. Our simulation results are pre-

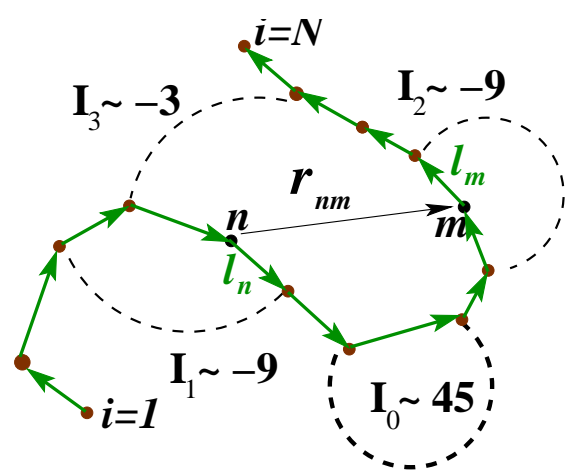

FIG. 1: Sketch of the bond-bond correlation $\left\langle l_{n} \cdot l_{m=n+s}\right\rangle$ of the bond vectors $l_{i}=r_{i+1}-r_{i}, r_{i}$ being the position vector of monomer $i$. The bond-bond correlation function is defined as the first Legendre polynomial $P_{1}(s)=\left\langle l_{n} \cdot l_{n+s}\right\rangle_{n} / l^{2}$ over all possible bond pairs with curvilinear distance $s=m-n \geq 0$ normalized by the mean squared bond length $l^{2}=\left\langle l_{n}^{2}\right\rangle$. The typical size of the corresponding chain segments is $R(s)=$ $\left\langle r_{n m}^{2}\right\rangle_{n}^{1 / 2}$ with $r_{n m}=r_{m=n+s}-r_{n}$. The dashed lines show the four relevant graphs of the analytical perturbation calculation. The numerical factors indicate the relative weights of the leading $1 / s^{3 / 2}$ contributions to $P_{1}(s)$.

sented first and discussed together with simple scaling arguments. We focus on the scale free limit and finite chain size effects $(s \rightarrow N)$ are considered more briefly. The analytical calculation (summarized graphically in Fig. (1) is outlined at the end of paper.

Two standard simulation methods for coarse-grained polymer chains 4] have been used to equilibrate long flexible polymer chains for which the bond-bond correlation functions presented in Figs. 2 , 3 and 4 below have been computed. The body of our data comes from the "bond fluctuation model" (BFM) - a lattice Monte Carlo scheme where a monomer occupies 8 lattice sites (i.e., the 
volume fraction is $8 \rho$ ) and the bonds $l_{i}$ between adjacent monomers can vary in length and direction, subject only to excluded volume constraints and entanglement restrictions [7, 8]. Using a mixture of local, slithering snake and double-bridging moves [7, 9] we have created ensembles with chain lengths ranging up to $N=2048$ for densities between $\rho=0.00195 / 8$ to $\rho=0.5 / 8$ contained in large periodic boxes of linear size $L=512 \gg R_{e}$ which allows us to eliminate finite-size effects. As can be seen in Fig. 22 we have also studied single chains up to $N=32768$ in an infinite non-periodic box $(\rho=0)$ to characterize properly the dilute reference point. Additional molecular dynamics simulations of the bead-spring model discussed in [7] have been performed to check explicitly that our results are not caused by lattice artifacts. As shown in Fig. 4 for one example with $N=256$ and $\rho=0.83$, identical behavior (although with reduced statistics) has been found.

Bond-bond correlation functions for different densities are presented in Fig. 2 where we focus first on the behavior of long chains $(s \ll N)$. In the dilute limit $(\rho \rightarrow 0)$ the athermal BFM chains are well fitted by $R(s)=b_{0} s^{\nu}, \nu=0.588$ being Flory's good solvent exponent and $b_{0} \approx 3.0$. The power law slope with $\omega=\omega_{0} \equiv 2-2 \nu \approx 0.824$ and $c_{a}=\nu(2 \nu-1)\left(b_{0} / l\right)^{2}$ observed in this limit (dashed line) is in fact expected [10] from the formula

$$
\left\langle l_{n} \cdot l_{m}\right\rangle=\left\langle\partial_{n} r_{n} \cdot \partial_{m} r_{m}\right\rangle=-\frac{1}{2} \partial_{n} \partial_{m}\left\langle r_{n m}^{2}\right\rangle
$$

expressing $P_{1}(s)$ as the second derivative of $R(s)$. It is crucial that $2 \nu>1$ and, hence, $\omega_{0}<1$, i.e. the integral over the correlation function is dominated by its upper bound. Otherwise, $\omega$ could not be governed by the exponent $\nu$ describing the asymptotic chain size. Interestingly, it follows also directly from Eq. (11) for ideal gaussian chains $\left(2 \nu \rightarrow 1: c_{a} \rightarrow 0\right)$ without additive power law corrections that $P_{1}(s)$ must decrease stronger than any power law, i.e. exponentially.

Coming back to Fig. 22 we note that also for finite densities $P_{1}(s)$ coincides with the dilute power law for small curvilinear distances $s$ where each chain segment interacts primarily with itself. At larger $s \gg g(\rho)$ where the chains overlap and form a "melt of blobs" the correlation function decreases much faster, however not exponentially as one might expect, but with a second power law regime with $\omega \approx 3 / 2$ (bold lines). For the BFM the density $\rho=0.5 / 8$ corresponds to a polymer melt since the excluded volume size becomes of order of the average bond size [7]. We observe indeed that the dilute slope disappears in the melt limit and the correlation function can be fitted (bottom bold line) over nearly two orders of magnitude by

$$
P_{1}(s)=c_{a} s^{-\omega} \text { with } \omega=3 / 2, c_{a}=\frac{\sqrt{6 / \pi^{3}}}{4 \rho l^{3}}
$$

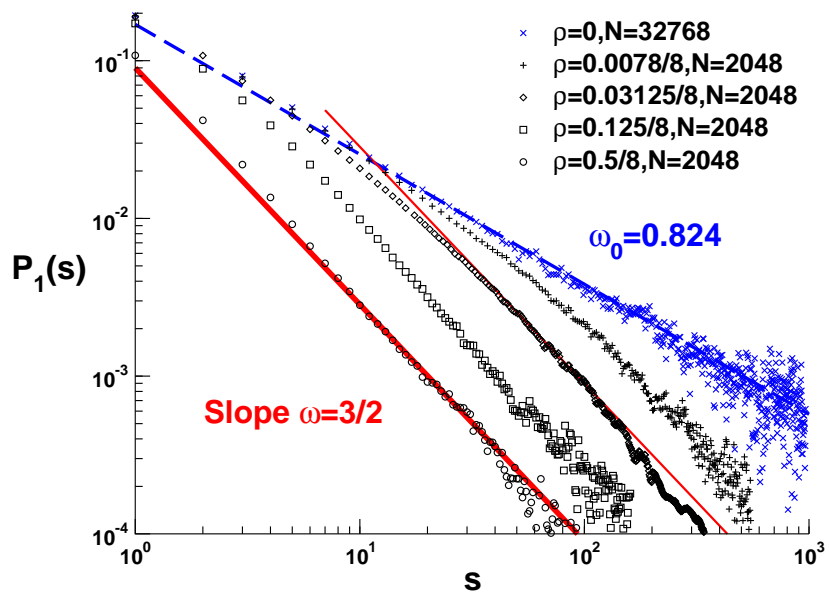

FIG. 2: The bond-bond correlation function $P_{1}(s)$ for BFM systems of different densities as indicated in the legend. The two lines indicate power laws corresponding to the asymptotic regime for dilute ( $\omega_{0} \approx 0.824$, dashed line) and dense ( $\omega=3 / 2$, bold lines) solutions, respectively. The observation of the second power law regime is the central result of this work. For systems in the so-called semidilute regime where the excluded volume is sufficiently large both exponents can be seen. The curvilinear distance at the crossover corresponds to the number of monomers per blob $g(\rho)$.

where we anticipate the analytical prediction for the asymptotic scale free limit. Considering that all parameters are known the agreement is excellent although a slightly better fit could be obtained by accepting a larger exponent. Obviously, the chain statistics in the semidilute and melt regime must become in leading order gaussian, i.e. the integral over Eq. (11) must be dominated by the lower bound in agreement with the finding $\omega>1$. In fact, Eq. (2) is consistent with

$$
R(s)^{2}=b^{2} s-\frac{2 c_{a} l^{2}}{(\omega-1)(2-\omega)} s^{2-\omega}
$$

with $b$ being the statistical segment length [2, 4]. As long as $\omega<2$, the correction term (being due to an upper integration bound) contributes a negative, but ever decreasing contribution to the chain distance, i.e., $R(s)^{2} / s$ approaches $b^{2}$ from below. Interestingly, Eq. (3) shows that the computation of $P_{1}(s)$ allows to highlight directly corrections to gaussian behavior which can be blurred by the large ideal term if moments of spatial distances are considered.

The scaling of $P_{1}(s)$ and $c_{a}$ with density are analyzed in Fig. 3] Following the classical density crossover scaling [1] we take as natural scaling variable $x=s / g(\rho)$ and

$$
P_{1}(s) g(\rho)^{\omega_{0}}=f(x) \sim\left\{\begin{array}{ll}
x^{-\omega_{0}} & , x \ll 1 \\
x^{-\omega} & , x \gg 1
\end{array} .\right.
$$

This is well borne out by the data. To be specific, we have used $g(\rho) \propto \rho^{-1 /(3 \nu-1)} \quad 12$ although the power law form cannot hold strictly for large densities where the 


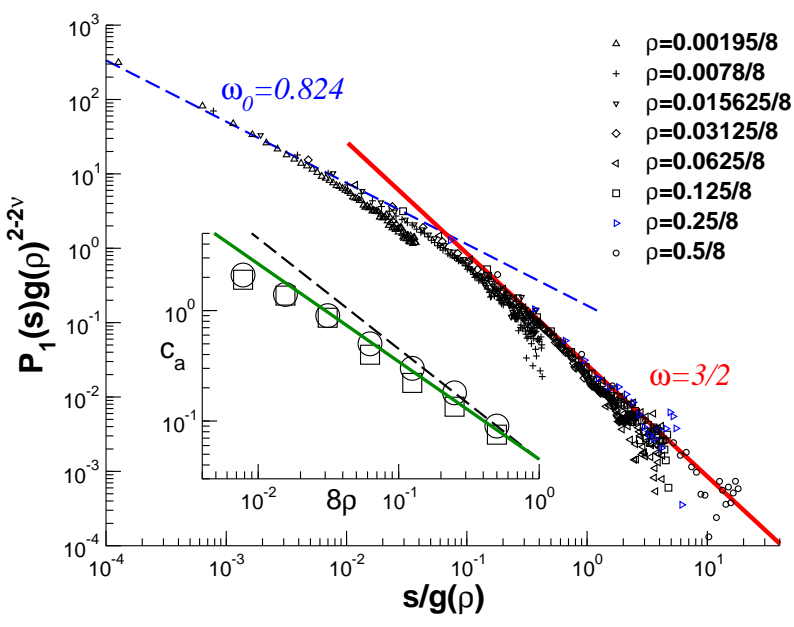

FIG. 3: Crossover scaling for $P_{1}(s)$ for the BFM $(N=2048)$ for different densities. In the main panel, the rescaled bondbond correlation function $f(x)=P_{1}(s) g(\rho)^{\omega_{0}}$ is plotted versus the natural scaling variable $x=s / g(\rho)$ assuming $g(\rho) \propto$ $\rho^{-1 /(3 \nu-1)}$. Note that for large $s / N$ the final cut-off (see Eq. (5)) becomes visible. In the inset we explicitly verify the density dependence of the amplitude $c_{a}(\rho)$ obtained from $P_{1}(s)$ (spheres) and $R(s)$ (squares) using Eq. (2) and Eq. (3), respectively. The prediction for the melt regime, Eq. (2), is indicated by the dashed line, the scaling $c_{a} \sim g(\rho)^{\omega-\omega_{0}} \sim$ $\rho^{-0.885}$ for the semidilute regime by the bold line.

semidilute blobs become too small and the assumed density dependence must be a crude estimate. Considering this, the scaling collapse for all densities (obtained without any arbitrary shift parameter) is far from obvious and quite satisfactory. The amplitude $c_{a}(\rho)$ of the nongaussian corrections has been fitted directly from $P_{1}(s)$ and $R(s)$ assuming $\omega=3 / 2$. As can be seen in the inset, the data compare rather well with the prediction for the semidilute regime $c_{a} \sim g(\rho)^{\omega-\omega_{0}} \sim \rho^{-0.885}$.

In the following we concentrate on dense melts. In Fig. 4 chain length effects are discussed at constant density. Data from the bead-spring model have also been included to demonstrate the universality of the result. To collapse this correlation function on the BFM data it has been vertically shifted by the ratio of the amplitudes $c_{a}$ calculated from Eq. (2) for BFM and bead-spring model. As can be seen for $N=16$, exponentials are compatible with the data of short chains. (This might explain how the power law scaling has been overlooked in previous numerical studies since good statistics for large chains $(N>1000)$ has only become available recently.) However, it is clearly shown that $P_{1}(s)$ approaches systematically the scale free asymptote with increasing $N$. The departure from this limit is fully accounted for by the theory if chain end effects are carefully considered (dashed lines). Generalizing Eq. (2), perturbation theory yields

$$
P_{1}(s)=c_{a} s^{-3 / 2} a(x)+c_{b} s^{-1 / 2} b(x)
$$

where we have set $x=\sqrt{s / N}$ and $c_{b}=4 c_{a} / N$ and

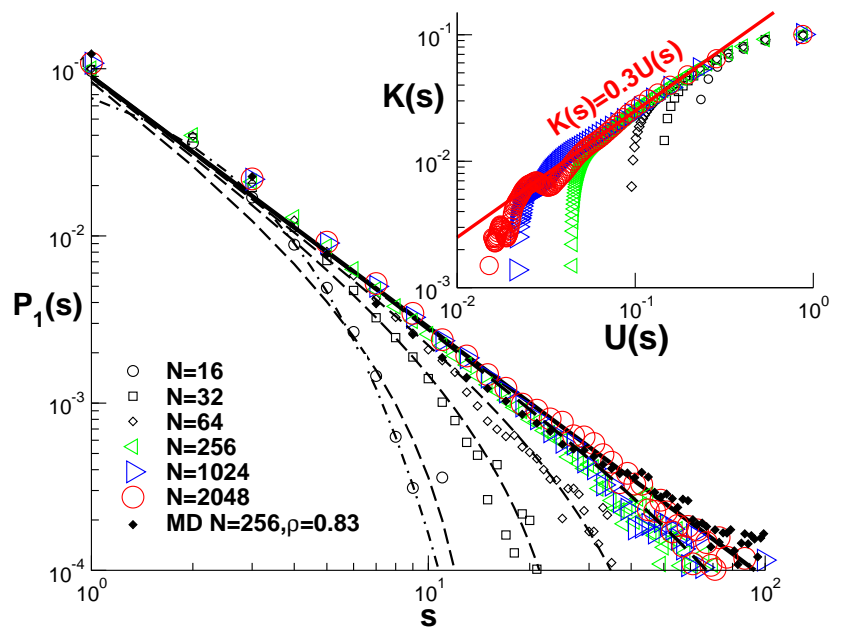

FIG. 4: Bond-bond correlation function versus $s$ at melt densities for different $N$ for the BFM $(\rho=0.5 / 8)$ and the beadspring model $(\rho=0.83)$ for $N=256$. The bold line indicates Eq. (2) with $c_{a} \approx 0.09$ for long BFM chains. The dasheddotted curve $P_{1}(s) \approx \exp (-s / 1.5)$ shows that exponential behavior is compatible with the small $N$ data. The dashed lines correspond to the complete theoretical prediction Eq. (5) for finite chain lengths $N=16,32,64$ and 256 (from left to right). Considering that the theory does not allow for any free fitting parameter the agreement is good. In the inset we check directly the recursion relation Eq. (6) by plotting $K(s)$ versus $U(s)=s / R(s)^{3} \rho$ (same symbols as in main figure). The proportionality of $K(s)$ and $U(s)$ is well confirmed (bond line) by our data for $1 \ll s \ll N$.

defined the functions $a(x)=b(x)\left(1+3 x+x^{2}\right)$ and $b(x)=(1-x)^{2} /(1+x)$. For $x \ll 1$ only the first term contributes: however, as $s \rightarrow N$ both terms become of similar magnitude. Both contributions to the correlation function vanish rigorously in this limit, and $P_{1}(s) \approx(1-x)^{2}$.

We outline now very briefly how Eqs. (21) and (51) have been obtained using perturbation calculation [13]. Closely following Edwards [2] we first determine $\left\langle r_{n m}^{2}\right\rangle \approx$ $\left\langle r_{n m}^{2}\right\rangle_{0}\left(1+\langle U\rangle_{0}\right)-\left\langle r_{n m}^{2} U\right\rangle_{0}$ and then, using Eq. (1), the bond-bond correlations. Here, $\langle\ldots\rangle_{0}$ denotes the average for the distribution function of the unperturbed ideal chain and $U=\int_{0}^{N} d k \int_{0}^{k} d l v_{1}\left(r_{k l}\right)$ the effective perturbation potential. The simplest approximation [2] for the pair interaction potential in Fourier space is $v_{1}(q)=v q^{2} /\left(q^{2}+\xi^{-2}\right)$ with $v$ being the bare excluded volume parameter and $\xi=\left(l^{2} / 12 \rho v\right)^{1 / 2}$ the screening length. This is sufficient for calculating the scale free regime (Eq. (2)). The graphs which contribute to $\left\langle l_{n} \cdot l_{m}\right\rangle$ and their relative weights are indicated in Fig. 1] Note that the interactions described by the strongest graph $I_{0}$ align the bonds $l_{n}$ and $l_{m}$ while the others tend to reduce the effect. More care is needed to describe properly the finite chain size corrections Eq. (5), and the Padé approximation for the intrachain structure factor must be used 
here. It can be readily shown that the different integrals are always dominated by long wavelength physics where $v_{1}(q) \rightarrow q^{2} l^{2} / 12 \rho \sim v^{0}$. Therefore, the coefficients $c_{a}$ and $c_{b}$ of Eqs. (2) and (5) do not depend on local interaction parameters, such like $v$ 14].

Since these calculations are lengthy we present a simple scaling argument for the $\omega$ exponent which captures the central physical idea [5]. As suggested by Eq. (3) a direct measure for the non-gaussian corrections can be defined by $K \equiv\left(R^{2}(2 s)-2 R^{2}(s)\right) /\left(2 R^{2}(s)\right) \approx c_{a} s^{1-\omega}$ where we compare the size of a segment of length $2 s$ with the size of two segments of length $s$ joint together. Equivalently, this can be read as a measure for the swelling of a chain where initially the interaction energy $U$ between the two halves has been switched off. Obviously, $K$ must be a functional of $U$ and $K(U=0)=0$. Perturbation theory tells us that the lowest order of the expansion of $K$ in $U$ must be linear. What is still missing is an estimate for the scaling of the interaction energy between the segments. For incompressible melts it is well known 1] that the energy penalty for joining two chains of length $N$ is set by the depth of the correlation hole, $U(N) \approx N / R(N)^{d} / \rho \sim N^{1-d \nu}$ [5, 11]. This generalizes to arbitrary $s$. Hence, in $d=3$ the interaction energy is small $\left(U(s) \sim 1 / s^{1 / 2}\right)$ which finally justifies the perturbation calculation. Taking everything together we obtain $\omega=d \nu=3 / 2$ and the important recursion relation

$$
K(s)=\frac{R^{2}(2 s)-2 R^{2}(s)}{2 R^{2}(s)} \approx U(s) \equiv \frac{s / R(s)^{d}}{\rho} .
$$

The recursion relation provides a very compact and entirely self-consistent description of dense polymer melts $(d>2)$. To lowest order it leads to Eqs. (2) and (3). This shows that while the chain configurations must be gaussian to leading order, corrections do necessarily occur even for very long chains. Eq. (6) has been directly validated in the inset of Fig. 4 where we have plotted $K(s)$ versus $U(s)$. The predicted linearity is well confirmed for large segments $(s \gg 1)$. The recursion relation being independent of the total chain length $N$ does not capture the scaling for very large segments. Hence, the breakdown of the scale free behavior for $K(U)$ (inset) and $P_{1}(s)$ (main figure) are both due to the same finite chain size effect.

In summary, we have shown that long range correlations exist in polymer melts and semidilute solutions due to both chain connectivity and incompressibility. Their most striking effect is the power law asymptote for the bond-bond correlation function allowing a direct numerical test of the non-gaussian corrections suggested by the self-consistent recursion relation Eq. (6). An important consequence of this work arises for an experimentally relevant quantity, the static structure factor $S(q)$. In fact, simulation and theory show distinct non- monotoneous behavior of $q^{2} S(q)$ vs. $q$ (Kratky plot) due to the non-gaussian corrections. This suggests a possible route for experimental verification and is cause of serious concerns with regard to the standard operational definition and measure of the persistence length from the assumed Kratky plateau. Finally, we point out that the physical mechanism which has been sketched above is rather general and should not be altered by details such as a finite persistence length - at least not as long as nematic ordering remains negligible. This is in fact confirmed by preliminary and on-going simulations.

We thank J.-L. Barrat and K. Binder for useful discussions and acknowledge financial support from the LEA and the ESF SUPERNET programme as well as computational resources by IDRIS, Orsay.

* Electronic address: jwittmer@ics.u-strasbg.fr

[1] P.-G. De Gennes, Scaling Concepts in Polymer Physics, (Cornell University, Ithaca, N.Y., 1979).

[2] M. Doi and S. F. Edwards, The Theory of Polymer Dynamics (Clarendon Press, Oxford, 1986).

[3] P. J. Flory, Statistical Mechanics of Chain Molecules, (Oxford University Press, New York, 1988).

[4] M. Muthukumar, S. F. Edwards, J. Chem. Phys. 76, 2720 (1982).

[5] A.N. Semenov, A. Johner, Eur. Phys. J. E. 12, 469 (2003).

[6] O. Kratky, G. Porod, Rec. Trav. Chim. 68, 1106 (1949).

[7] J. Baschnagel, J.P. Wittmer, H. Meyer, Monte Carlo Simulation of Polymers: Coarse-Grained Models, in Computational Soft Matter: From Synthetic Polymers to Proteins edited by N. Attig et al. (NIC Series, Volume 23, Jülich, 2004), pp. 83-140.

[8] I. Carmesin and K. Kremer, Macromolecules, 21, 2819 (1988); H. P. Deutsch and K. Binder, J. Chem. Phys., 94, 2294 (1991); W. Paul et al, J. Phys. II, 1, 37 (1991).

[9] R. Auhl et al, J. Chem. Phys. 119, 12718 (2003).

[10] L. Schäfer, K. Elsner, Eur. Phys. J. E 13, 225 (2004).

[11] Interestingly, $U$ does not depend explicitly on the excluded volume parameter $v$. The scaling of $U(r, N)$ has been verified directly by measuring the pair correlation function $p(r)$ of the chain center of masses and using $\exp (-U(r, N)) \approx p(r) / p(\infty)$.

[12] The prefactor has been obtained from the scaling of $R(s)$ for $\rho<0.125 / 8$.

[13] A similar description has been also obtained for polydisperse living polymers using the polymer-magnetic analogon. This will be presented elsewhere.

[14] Contrary to this, local interaction parameters do modify the statistical segment length $b$ 2, 4]. Interestingly, the generic behavior of the bond-bond-correlation function does not change under systematic coarse-graining $\lambda$ neighboring bonds. Our simulation results confirm again the expected scaling $P_{1}(s ; \lambda) \approx \frac{1}{(\rho / \lambda)\left(l \lambda^{1 / 2}\right)^{3}}(\lambda / s)^{\omega=3 / 2}$ for long chains. 